\title{
Sewage from Airplanes Exhibits High Abundance and Diversity of Antibiotic Resistance Genes
}

\section{Hess, Stefanie}

2019-12-03

Hess , S , Kneis , D , Osterlund , T , Li , B , Kristiansson, E \& Berendonk , T U 2019 , ' Sewage from Airplanes Exhibits High Abundance and Diversity of Antibiotic Resistance

Genes ' , Environmental Science \& Technology , vol. 53 , no. 23 , pp. 13898-13905 . https://doi.org/10.1021/acs.est.

http://hdl.handle.net/10138/326230

https://doi.org/10.1021/acs.est.9b03236

unspecified

acceptedVersion

Downloaded from Helda, University of Helsinki institutional repository.

This is an electronic reprint of the original article.

This reprint may differ from the original in pagination and typographic detail.

Please cite the original version. 


\title{
Sewage from airplanes exhibits high abundance and diversity of antibiotic resistance genes
}

\author{
Heß, Stefanie ${ }^{\mathrm{b} *}$, Kneis, David ${ }^{\mathrm{a}, \mathrm{f}}$, Österlund, Tobias ${ }^{\mathrm{c}}$, Li, Bing ${ }^{\mathrm{d}}$, \\ Kristiansson, Erik ${ }^{\mathrm{c}, \mathrm{e}}$, Berendonk, Thomas U. ${ }^{\mathrm{a}}$ \\ a Institute of Hydrobiology, TU Dresden, Germany \\ b Dept. of Microbiology, University of Helsinki, Finland \\ c Mathematical Sc. Dept., Chalmers University of Technology, Gothenburg, Sweden \\ d Division of Energy and Environment, Graduate School at Shenzhen, Tsinghua University, China \\ e Centre for Antibiotic Resistance Research (CARe), University of Gothenburg, Sweden \\ f Helmholtz-Centre for Environmental Research, Magdeburg, Germany \\ * Corresponding author: $\quad$ stefanie.hess@helsinki.fi
}

\begin{abstract}
1 Abstract
2 Airplane sanitary facilities are shared by an international audience. We hypothesized 3 the corresponding sewage to be an extraordinary source of antibiotic resistant bacteria 4 (ARB) and resistance genes (ARG) in terms of diversity and quantity. Accordingly, 5 we analyzed ARG and ARB in airplane-borne sewage using complementary approaches: 6 metagenomics, quantitive PCR, and cultivation. For the purpose of comparison, we also 7 quantified ARG and ARB in the inlets of municipal treatment plants with and without 8 connection to airports. As expected, airplane sewage contained an extraordinary rich 9 set of mobile ARG and the genes' relative abundances were mostly increased compared 10 to typical raw sewage of municipal origin. Moreover, combined resistance against third 11 generation cephalosporins, fluorochinolones and aminoglycosides was unusually common 12 (28.9\%) among E. coli isolated from airplane sewage. This percentage exceeds the one 13 reported for German clinical isolates by a factor of eight. Our findings suggest that 14 airplane-borne sewage can effectively contribute to the fast and global spread of antibiotic resistance.
\end{abstract}

Keywords: antibiotic resistance, airplane, sewage, wastewater, diversity 


\section{Introduction}

Since their discovery in the 1940s, antibiotics saved millions of lives, but due to the global spread of resistance genes these drugs rapidly lose their activity. This threatens the very core of modern medicine by limiting the means to effectively cure bacterial infections (World Health Organization, 2015). In the era of globalization and high mobility, pathogenic strains carrying antibiotic resistance genes (ARG) are spreading quickly and globally (Nordmann et al., 2011). In the endeavor to decelerate or stop the spread of antibiotic resistance, it is necessary to identify the hotspots and pathways of ARG dissemination.

Airports are places where sewage of people from different parts of the world is collected and, after treatment, released in the local aquatic environment. It is thus reasonable to assume that airports serve as an entrance for ARG which are endemic in specific parts of the world while being rare or absent in the flights' country of destination. This should be reflected in a particularly high diversity of ARG in airplane-borne sewage as compared to conventional municipal wastewater (hypothesis $\mathbf{1}$ ).

The prevalence of antibiotic resistant bacteria (ARB) is known to be linked with veterinary and human antibiotic use (World Health Organization, 2014). Specifically, Forslund et al. (2013) found the resistance potential in human guts to be positively correlated with country-specific antibiotic use. In Germany, antibiotic consumption is relatively low compared to, e.g., China, India, or the USA (Van Boeckel et al., 2014) and it is also lower than in many member states of the EU (European Surveillance of Antimicrobial Consumption Network, 2017). Consequently, airplane-borne sewage was hypothesized to contain ARB and ARG in elevated abundances as compared to standard municipal sewage generated by the local population (hypothesis 2 ).

The two hypotheses related to the diversity and abundance of ARG were tested by the complementary approaches of metagenome sequencing, quantitative PCR (qPCR), and cultivation, thereby providing a comprehensive picture of antimicrobial resistance in studied systems. Specifically, the strength of the metagenomics approach lies in its capability to detect and quantify the full spectrum of ARG with the downside of limited sensitivity. Quantitative PCR, on the other hand, allows even rare genes and gene variants to be quantified since the target sequences are specifically amplified. However, qPCR necessarily has a narrow focus dictated by the chosen primers. Finally, susceptibility tests provide the only means to study phenotypic antibiotic resistance, including multi-resistance. Unlike the other techniques, cultivation covers a small part of the microbial community only.

In this study, any of the three approaches was used to quantify antibiotic resistance in untreated sewage collected from (1) airplane tanks and (2) the inlets of municipal wastewater treatment plants (WWTP). The set of WWTP was chosen such that some of the plants received wastewater from the nearest airport while others did not. The latter distinction was made to test whether the hypothesized peculiarities of airplane-borne sewage are still noticeable after mixing with "conventional" municipal wastewater.

\section{Material and methods}

\section{$2.1 \quad$ Sampling}

Eight grab samples of airplane-borne sewage were obtained from five different airports, three of which were sampled twice (with sufficient delay to ensure independence). Three of the airports had $>20$ million passengers p.a. in 2015 while the other two were of intermediate size ( $>10$ million passengers p.a.) or smaller. The sewage was gathered from vacuum trucks collecting the waste from multiple aircrafts. Since the aircraft's 
tanks are emptied on demand, each tank contains sewage from multiple flights. The samples thus represent pooled samples integrating over space and time.

Incoming sewage from WWTP having a connection to airports was collected at six different locations, two of which were visited twice (total number of eight samples; each being a $24 \mathrm{~h}$ composite). At the respective plants, airport-borne sewage was known to contribute at least $20 \%$ of the total inflow. These $20 \%$ represent a mixture of fecal material from airplane tanks with sewage from other airport facilities such as terminal buildings. Detailed data on mixing ratios and its temporal variation were unavailable to us. Surface runoff from airports is generally disposed separately from sewage not least because of the possible contamination with, e.g., de-icing agents.

Incoming sewage from WWTP without connection to airports was collected at six locations. At one of the locations, samples were taken from four different sewers and another plant was sampled multiple times (with sufficient delay to ensure independence). The total number of $24 \mathrm{~h}$ composite samples was 19 .

All samples were stored in $1 \mathrm{~L}$ sterile glass bottles at $4{ }^{\circ} \mathrm{C}$ and processed within $24 \mathrm{~h}$. Relevant meta-information is provided in Table S.1.

\subsection{Metagenomics}

DNA was extracted using the PowerWaterKit (MoBio, Vancouver, Canada; see Table S.1 for amount and quality of DNA). All samples were shotgun-sequenced on a MiSeq device $(2 \times 150 \mathrm{bp}$; GATC Biotech AG, Konstanz, Germany). Quality checking and trimming ( $\mathrm{q}=28$, minimum length $=100 \mathrm{bp}$ ) was performed using TrimGalore! (http: //www.bioinformatics.babraham.ac.uk/projects/trim_galore/). The preprocessed forward reads were then aligned to the latest (2019-03-05) resfinder data base (Zankari et al., 2012). Specifically, we relied on the python implementation of the resfinder script which is publicly available from the Center for Genomic Epidemiology (http: //www.genomicepidemiology.org/). The resfinder script attempts to match the nucleotide sequences contained in the samples with the sequences of known ARG as stored in the resfinder data base. Internally, the resfinder script calls the Basic Local Alignment Search Tool (blastn; http://blast.ncbi.nlm.nih.gov) to compare nucleotide sequences. blastn-reported alignments with e-values exceeding $10^{-10}$ were generally ignored to minimize the chance of false positive hits.

The set of unique ARG contained in a particular sample was established from the full list of resistance genes whose signatures matched with a nucleotide sequence from the sample. Technically speaking, the obtained list of hits was filtered to remove any duplicate gene identifiers. The resf inder data base holds information on a large number genes and variants, e.g. about 2000 of the registered ARG target beta-lactams. So as to focus on ARG diversity at a higher level, we generally truncated the genes' identifiers at the first underscore before establishing the unique set of ARG. For example, instead of counting the tetracycline resistance gene variants tet(M)_7_FN433596 and tet(M)_1_X92947 as individual instances, they were commonly registered as just tet (M). For the purpose of additional quality assurance, a particular gene was only registered as present if its signature was detected at least three times. Moreover, nucleotide sequences matching the signatures of multiple ARG registered in the resfinder data base (ambiguous hits), were ignored altogether.

\subsection{Quantitative PCR}

Fourteen ARG were selected for qPCR-based quantification in line with activities of international research consortia investigating antimicrobial resistance in aquatic environments. The set of ARG includes common and rare ones with a special focus on clinically 
relevant genes coding for carbapenemases (blatem, bla $a_{\mathrm{CTX}-\mathrm{M}-15}$, bla $\mathrm{CTX}_{\mathrm{CT}-32}$, bla $\mathrm{CMY}_{\mathrm{C}-2}$, bla OXA-48, bla OXA-58, bla KPC-3 $_{3}$, bla NDM-1 $_{1}$, blaviM-2, ermB, mecA, mcr1, tetM, sul1). All ARG as well as 16S rRNA genes were quantified following the same procedure as described in Heß et al. (2018). The relevant references for the primers of the additionally quantified ARG are the NORMAN network (www.norman-network.net) for bla TEM, and

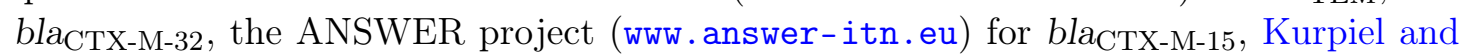
Hanson (2012) for bla CMY-2, Monteiro et al. (2012) for blaoxA-48, Hembach et al. (2017) for mcr1, and Peak et al. (2007) for tetM. Primer sequences can be found in Table S.2. For the 16S rRNA gene, sul1 and bla $\mathrm{CTX}-\mathrm{M}-32$ the pNORM plasmid designed by Ch. Merlin (University of Lorraine, France; www.norman-network.net) was used as standard. For the remaining genes, standards were created by cloning the respective $\mathrm{qPCR}$ amplicons into the pGEM-T vector (Promega, Madison, Wisconsin, USA).

The detection limit was $10^{2}$ copies per reaction for all genes. The efficiency of these assays was between 0.9 and 1 with $\mathrm{R}^{2}>0.997$ for all the runs. All genes were quantified in duplicates with a standard deviation of the cycles $<0.2$.

\subsection{Standardization of data and community analysis}

In the context of this study, the diversity and abundance of ARG was to be compared across samples of varying composition and dilution (toilet waste, municipal wastewater), hence, standardization was required. With regard to ARG abundances, we adopted the common practice of dividing absolute ARG counts by the corresponding number of $16 \mathrm{~S}$ rRNA copies (see, e.g. Pärnänen et al., 2019). The resulting numbers are referred to as relative abundances. We applied the same standardization also to metagenomics-based information on ARG diversity. Specifically, the diversity of resistance genes was expressed as the number of unique ARG per 1000 copies of $16 \mathrm{~S}$ rRNA genes.

The calculation of relative ARG abundances from qPCR-based data involved a qPCRbased quantification of $16 \mathrm{~S}$ rRNA genes (see Table S.2 for primer). Likewise, metagenomicsbased information on ARG was standardized using metagenomics-based estimates on the abundance of $16 \mathrm{~S}$ rRNA genes. The latter was extracted from the nucleotide sequences using METAXA2 (Bengtsson-Palme et al., 2015b, version 2.1.3).

The number of $16 \mathrm{~S}$ rRNA gene copies per bacterial cell is known to vary between taxonomic groups. Consequently, a comparison of relative ARG abundances (or ARG diversities) requires that the respective microbial communities are similar in terms of taxonomic composition or, at least, with regard to the community-weighted average number of 16S rRNA gene copies per cell. We employed METAXA2 to infer information on the composition of the microbial community based on 16S rRNA. Finally, we relied on the rrnDB data base (Stoddard et al., 2015) to compute for all metagenomics-samples the expected average number of $16 \mathrm{~S}$ rRNA copies per bacterial cell taking into account taxonomic groups and their proportions. The matching of taxonomic items reported by METAXA2 with items registered in $\operatorname{rrnDB}$ was successful at family level in $86 \%$ of the cases, at genus level in $54 \%$ of the cases and at species level in $10 \%$ of the cases.

\subsection{Bacteria isolation and susceptibility testing}

Escherichia coli was chosen as a model organism because it is widely considered as a fecal indicator and resistance levels of E. coli from different origins are well documented (e.g. European Centre for Disease Prevention and Control, 2017; Osińka et al., 2017; Rosas et al., 2015). E. coli is furthermore a potential pathogen and its harboring of resistance genes can thus directly impact human health.

To obtain at least $24 \mathrm{E}$. coli isolates from each sample, suitable dilutions were plated on mFC agar (Carl Roth, Karlsruhe, Germany). After $18 \pm 2 \mathrm{~h}$ of incubation at $44^{\circ} \mathrm{C}$ 
blue colonies were streaked on Brilliance agar (Oxoid, Wesel, Germany) and grown overnight at $37^{\circ} \mathrm{C}$ to obtain pure cultures. To identify the isolates as E. coli, colony PCR was performed as in Heß et al. (2018) to amplify a species specific fragment of the ycc $T$ gene.

In total, 1140 E. coli isolates (Table S.1) were tested against 24 antibiotics which are commonly used to treat the respective infections (187 isolates from airplanes, 161 and 362 isolates from the inflow of municipal treatment plants with and without connection to an airport). The tests followed the EUCAST guidelines (agar diffusion test; www. eucast.org) with Escherichia coli ATCC 25922 as a quality control. Applying the clinical breakpoints defined by EUCAST, the isolates were classified as resistant or susceptible (intermediary was counted as susceptible).

\subsection{Statistics}

Data analysis was conducted with R 3.4.3 (R Core Team, 2017). Relative ARG abundances in samples of different origin were compared using the Welch test (t.test ()) with log-transformed data. A non-parametric rank sum test (wilcox.test ()) was used to test for differences in ARG richness. Proportion data were tested for significant differences using Fisher's exact test (fisher.test ()). In case of multiple tests, p-values were conservatively adjusted with the default p.adjust () method Holm (1979). p-values were marked with asterisks according to the usual convention where ${ }^{*}$ indicates $p \leq 0.05$, ** denotes $p \leq 0.01$ and ${ }^{* * *}$ corresponds to $p \leq 0.001$. Bootstrap confidence intervals (Fig. 5) were generated with boot() using ordinary resampling and $10^{4}$ replicates. Rarefaction curves (Fig. 3) were constructed from metagenomics data by evaluating an increasing number of sequences from the whole set of sequences available for a particular sample by means of sampling without replacement ( $R$ method sample). To minimize random effects, we considered the medians of five replicate rarefaction curves per sample. Statistical models fitted to empirical rarefaction curves (Fig. 3) take the structure of Eqn. 1 where $R$ represents the number of different ARG, $n$ denotes the number of analyzed sequences, and $R_{\text {inf }}$ and $h$ are free parameters, respectively, fitted with R's default optimizer (optim).

$$
R=R_{\text {inf }} \cdot\left(\frac{n}{n+h}\right)^{0.5}
$$

Based on the overall number of sequences in a sample and the corresponding number of $16 \mathrm{~S}$ rRNA copies, the variable $n$ was adjusted individually for each sample to pragmatically compensate for varying proportions of bacterial and non-bacterial DNA.

\section{Results}

\subsection{Characterization of samples}

In accordance with expectation, untreated wastewater sampled at WWTP inlets was generally more diluted as compared to the sewage derived from airplane tanks. This is reflected, for example, in the electric conductivity but also in the number of $16 \mathrm{~S}$ rRNA gene copies per volume (Table 1). In addition, airplane sewage exhibited higher values of $\mathrm{pH}$ which is likely due to an elevated concentration of soap residues.

The metagenomics-derived information on 16S rRNA fragments allowed for a deeper characterization of the samples in terms of the composition of the bacterial community. The latter was very similar in all samples taken at the inlets of WWTP whereas airplaneborne samples showed larger variations in the contribution of different bacterial orders (Fig. 1). Moderate contrasts were observed between samples of different origins in terms 
Table 1: Mean values of electric conductivity (EC), $\mathrm{pH}$, and the concentration of $16 \mathrm{~S}$ rRNA genes in samples of different origins. The corresponding information for individual samples can be found in Table S.1.

\begin{tabular}{lrrr}
\hline Origin & $\mathrm{EC}\left(\mathrm{mS} \mathrm{cm}^{-1}\right)$ & $\mathrm{pH}$ & 16S rRNA gene copies $\mathrm{mL}^{-1}$ \\
\hline Airplane & 10.1 & 8.9 & $1.6 \times 10^{9}$ \\
WWTP, with airport & 1.7 & 7.3 & $2.6 \times 10^{8}$ \\
WWTP, no airport & 1.1 & 7.3 & $2.9 \times 10^{8}$ \\
\hline
\end{tabular}

of taxonomic diversity. For example, the signatures of 6.5 bacterial families (median) were detected in $10^{5}$ nucleotide sequences obtained from airplane sewage. This compares to values of 4 and 3 for wastewater from WWTP with and without connection to airports, respectively. This fact is also visible in Fig. 1 which shows a greater evenness in the contribution of taxonomic groups for airplane-borne samples as compared to WWTP samples. Characteristic differences between samples of different origins were observed, e.g., for Aeromonadales and Campylobacterales (rare in airplane sewage but common in WWTP samples) as well as Enterobacterales and Erysipelotrichiales (rare in WWTP samples but highly abundant in some airplane samples).
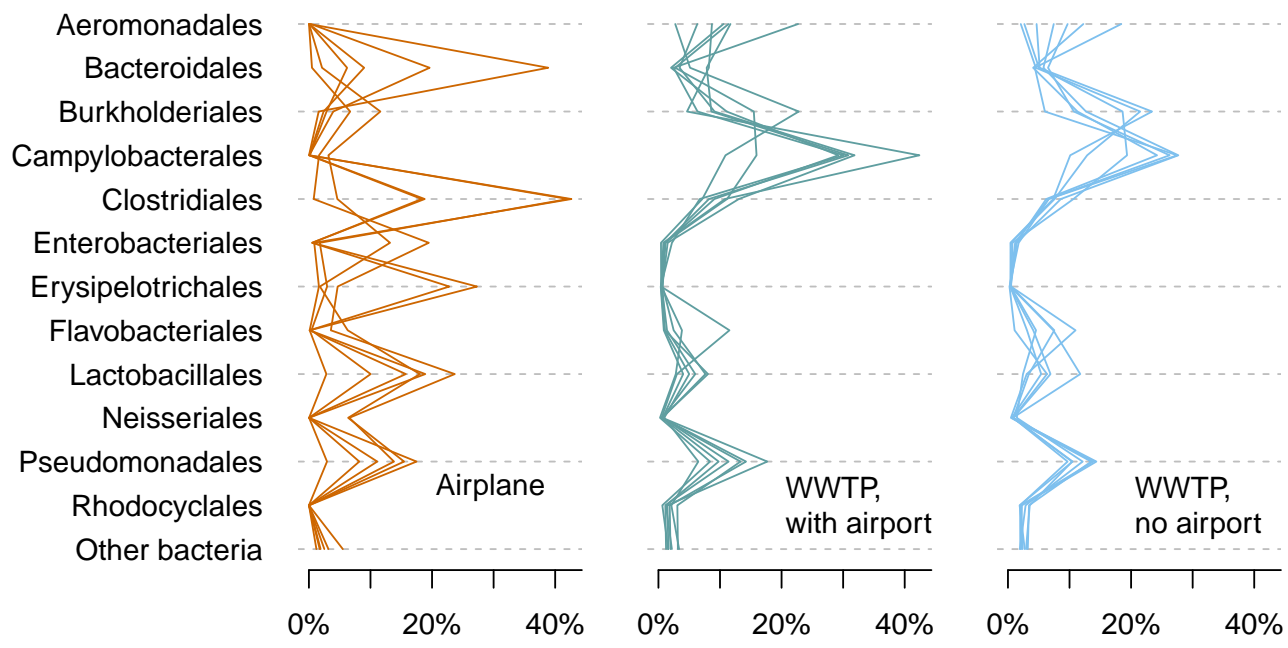

Figure 1: Percentage of $16 \mathrm{~S}$ rRNA fragments attributable to different bacterial orders. Lines depict individual samples. Bacterial orders which did not contribute at least $5 \%$ in any of the samples were dropped for the sake of clarity.

In spite of the differences in taxonomic composition, the expected number of $16 \mathrm{~S}$ rRNA genes per cell varied only little between sample of different origin. The merging of the metagenomics-based taxonomy data with information from the rrnDB data base resulted in a mean value of 4.9 copies of $16 \mathrm{~S}$ rRNA genes per cell for airplane-borne samples. The respective mean values for raw wastewater with and without connection to airports were 4.5 and 4.8 , respectively. The most extreme ratios observed for individual samples ranged between 4 and 5.5 and none of the differences in means fulfilled the criteria of statistical significance (ANOVA: $p>0.23$; all $p>0.13$ in post-hoc rank sum tests). In view of that, a standardization of ARG counts by the corresponding number of $16 \mathrm{~S}$ rRNA genes (see Sec. 2.4) was considered appropriate. In other words, it is very unlikely that marked shifts in the relative abundance of ARG, e.g. between samples of different origin, merely reflect dissimilarities of the respective bacterial communities. 


\subsection{Diversity of antibiotic resistance genes}

As outlined in Sec. 2.2, the diversity of antibiotic resistance genes was expressed as the number of unique ARG per 1000 copies of $16 \mathrm{~S}$ rRNA genes. That measure of diversity was found to be significantly increased in airplane sewage as compared to untreated sewage sampled from the inlets of municipal WWTP (Fig. $2 ; p<0.05$, Wilcoxon rank sum test). On average, a unique ARG was detected every 580 copies of $16 \mathrm{~S}$ rRNA genes in airplane sewage. In the WWTP samples, a unique ARG appeared every 750 copies of $16 \mathrm{~S}$ rRNA genes. WWTP samples were statistically similar in terms of ARG diversity regardless of whether the plant received sewage from an airport or not (blue vs. green boxes in Fig. 2).

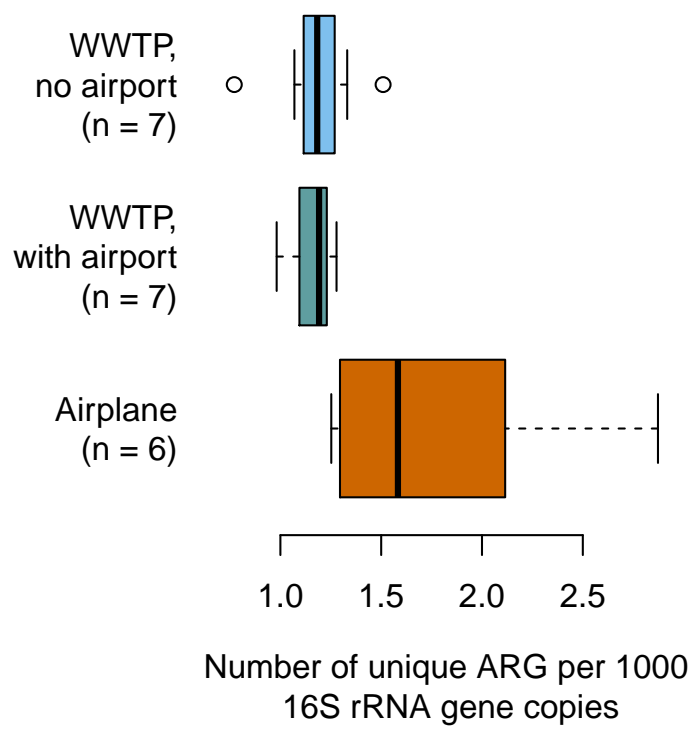

Figure 2: Diversity of antibiotic resistance genes in samples of untreated sewage collected from airplanes and WWTP with/without connection to airports. Whiskers extend to the most extreme data points not being classified as outliers.

The numbers on ARG diversity presented in Fig. 2 represent point estimates based on the number of sequences in each sample and the respective number of $16 \mathrm{~S}$ rRNA gene copies. In order to verify the robustness of these estimates, we computed rarefaction curves for all samples processed with metagenomics (Fig. 3). The latter confirm that samples of airplane sewage are likely to contain a greater number different ARG in a given amount of DNA as compared to untreated wastewater. For example, the average number of unique ARG per $5 \times 10^{6}$ sequences was about 110 in airplane-borne samples compared to about 85 in samples taken from the inlets of WWTP (Fig. 3). 


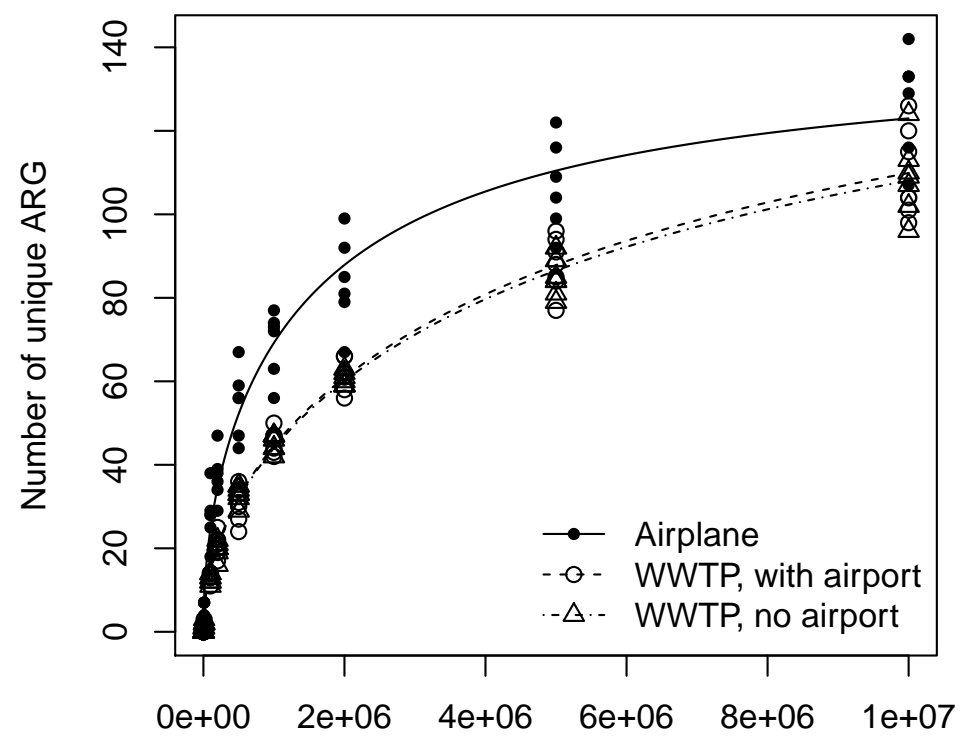

Adjusted number of sequences

Figure 3: Rarefaction curves relating the number of unique ARG to the number of analyzed sequences (adjusted for varying abundances of $16 \mathrm{~S}$ rRNA genes). Point symbols represent empirical estimates for individual samples. Statistical models of the form of Eqn. 1 (lines) were fitted to all points of a particular sample origin.

\subsection{Abundance of antibiotic resistance genes}

The metagenomics data indicate an increased relative abundance of ARG in airplane sewage as compared to sewage sampled at the inlets of WWTP (Fig. 4). The elevated prevalence in airplane-borne sewage was most pronounced for ARG directed against phenicols, sulphonamides, and tetracyclin $(p<0.01)$ as well as aminoglycosides and macrolides ( $p<0.05$, two-sided Welch test, conservatively adjusted for multiple testing). Contrary to the trend, ARG targeting colistin were more abundant in municipal sewage than in airplane sewage (value near limit of quantification). Generally, the samples taken at treatment plants with and without connection to airports (green and blue symbols in Fig. 4) did not exhibit significant differences in terms of relative abundances according to the Welch test.

The auxiliary qPCR-based analyses support the main outcome of the metagenomics approach depicted in Fig. 4. Specifically, the relative abundances of sul1 (sulphonamide) and tetM (tetracycline) resistance genes were significantly increased in airplane samples compared to samples taken at the inlets of WWTP (Table 2). The median relative abundances differ between the two sample origins by factors of 5 (sul1) and 18 (tetM), respectively.

With the exception of blaviM-2, differences in relative abundance between airplane and WWTP samples were not statistical significant for the remaining ARG (Table 2). Especially for the ß-lactamase genes, this finding is compatible with the metagenomic results (Fig. 4) according to which the total prevalence of genotypic ß-lactam resistance was only slightly increased in airplane-borne samples. 


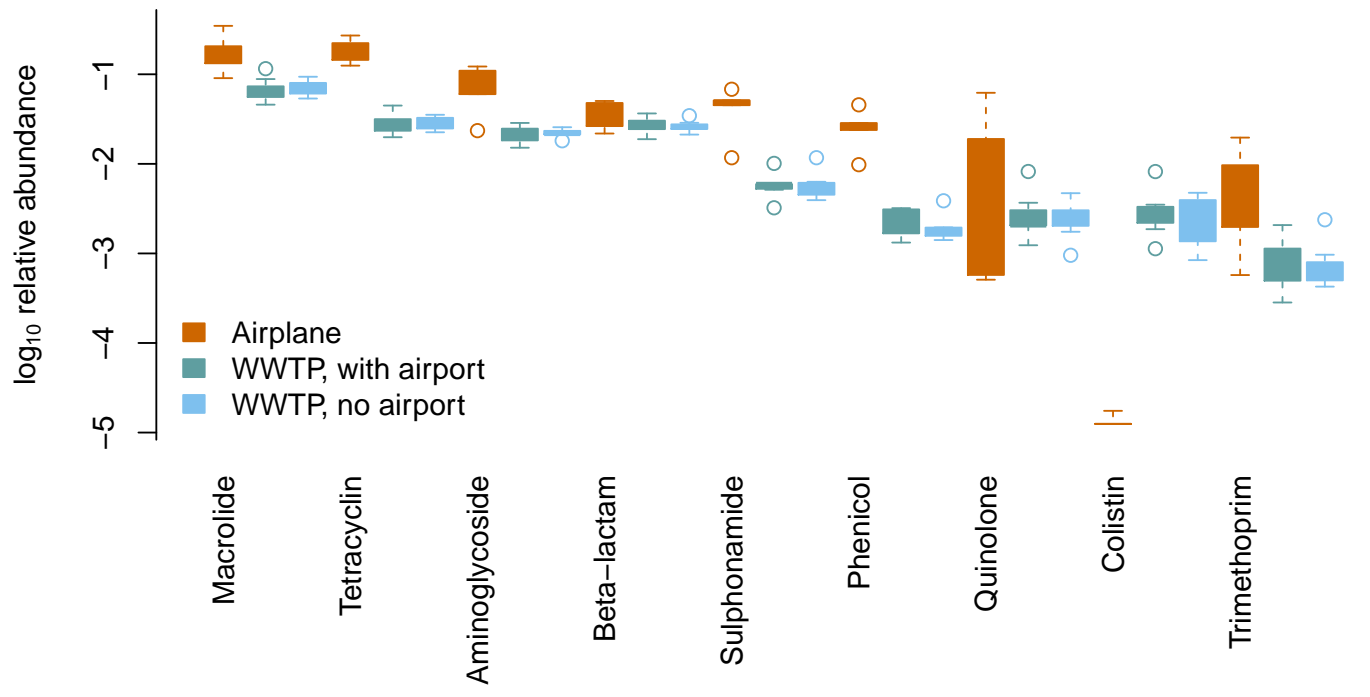

Figure 4: ARG copies per 16S rRNA gene copies in untreated sewage collected from airplanes and WWTP with/without connection to airports. The respective information on ARG and 16S rRNA genes is based on the metagenomics analysis. Genes were aggregated by target drug classes to reduce the complexity of information.

WWTP samples with and without contribution of airport sewage were also processed through qPCR. Like with the metagenomics approach, statistically significant differences between the two origins in terms of relative abundance could not be established (same set of ARG as in Table 2, results not shown).

Table 2: Relative abundance of resistance genes (ARG copies per 16S rRNA gene copies) in airplane sewage and the influent of wastewater treatment plants with connection to airports. Reported numbers are median values obtained by qPCR. Adjusted p-values refer to a two-sided Welch test.

\begin{tabular}{|c|c|c|c|c|}
\hline Target class & ARG & $\begin{array}{l}\text { Airplane } \\
(\mathrm{n}=8)\end{array}$ & $\begin{array}{l}\text { WWTP, with airport } \\
\qquad(\mathrm{n}=8)\end{array}$ & $\begin{array}{c}\text { adj. } \\
\text { p-value }\end{array}$ \\
\hline Colistin & $m c r 1$ & $2.8 \times 10^{-6}$ & $9.7 \times 10^{-6}$ & 0.81 \\
\hline MLSB & erm $\mathrm{B}$ & $2.0 \times 10^{-2}$ & $1.7 \times 10^{-2}$ & 1 \\
\hline ß-lactam & $b l a_{\mathrm{KPC}-3}$ & $1.6 \times 10^{-7}$ & $2.0 \times 10^{-7}$ & 1 \\
\hline ß-lactam & $b l a_{\text {OXA- } 48}$ & $6.5 \times 10^{-6}$ & $1.3 \times 10^{-3}$ & 0.33 \\
\hline ß-lactam & $b l a_{\text {OXA-58 }}$ & $1.6 \times 10^{-6}$ & $6.1 \times 10^{-4}$ & 0.22 \\
\hline ß-lactam & $b l a_{\mathrm{TEM}}$ & $1.1 \times 10^{-3}$ & $4.3 \times 10^{-4}$ & 1 \\
\hline ß-lactam & mecA & $2.7 \times 10^{-7}$ & $2.3 \times 10^{-7}$ & 1 \\
\hline B-lactam & $b l a_{\mathrm{CMY}-2}$ & $2.2 \times 10^{-4}$ & $1.8 \times 10^{-5}$ & 0.81 \\
\hline ß-lactam & $b l a_{\text {CTX-M-15 }}$ & $3.2 \times 10^{-4}$ & $4.5 \times 10^{-5}$ & 0.81 \\
\hline ßs-lactam & bla $a_{\mathrm{CTX}-\mathrm{M}-32}$ & $8.3 \times 10^{-4}$ & $1.3 \times 10^{-4}$ & 0.81 \\
\hline ßs-lactam & $b l a_{\mathrm{NDM}-1}$ & $1.5 \times 10^{-5}$ & $2.2 \times 10^{-5}$ & 1 \\
\hline ß-lactam & $b l a_{\mathrm{VIM}-2}$ & 0 & $1.4 \times 10^{-4}$ & $0.037^{*}$ \\
\hline Sulphonamide & sul1 & $4.7 \times 10^{-2}$ & $9.2 \times 10^{-3}$ & $0.019^{*}$ \\
\hline Tetracyclin & tet $\mathrm{M}$ & $2.4 \times 10^{-2}$ & $1.3 \times 10^{-3}$ & $9.4 \mathrm{e}-05^{* * *}$ \\
\hline
\end{tabular}




\subsection{Phenotypic resistance in Escherichia coli}

The highest percentage of resistant E. coli isolates was generally detected in airplane samples (Table 3). The difference in proportions between airplane and WWTP samples was statistically significant for 18 out of 24 antibiotics. Some Odds ratios reached values around 40, namely for two cephalosporins (cefotaxime, cefuroxime) and one of the fluoroquinolones (ciprofloxacin). At the same time, the influent of treatment plants receiving sewage from airports did not exhibit elevated proportions of resistant E. coli when compared to treatment plants that do not receive such input ( $\mathrm{p}$-values generally $>0.15$, results not shown).

Table 3: Antibiotic resistance among E. coli isolated from airplane sewage and the inlet of WWTP with connection to airports. Odds ratios (OR) greater than one indicate a higher level of resistance in the airplane samples compared to WWTP samples. pvalues refer to Fisher's exact test. SXT stands for the combination of trimethoprim and sulfamethoxazole also known as co-trimoxazole.

\begin{tabular}{lrrrr}
\hline Antibiotic & Airplane & WWTP, with airport & OR & adj. p-value \\
\hline Ampicillin & $125: 187$ & $21: 161$ & 13.3 & $2.9 \mathrm{e}-24^{* * *}$ \\
Amoxicillin-CA & $65: 187$ & $8: 161$ & 10.1 & $1.3 \mathrm{e}-11^{* * *}$ \\
Piperacillin & $21: 187$ & $5: 161$ & 3.9 & $0.032^{*}$ \\
Ticarcillin & $55: 187$ & $4: 161$ & 16.3 & $1.3 \mathrm{e}-11^{* * *}$ \\
Cefepime & $7: 187$ & $0: 161$ & Inf & 0.099 \\
Cefotaxime & $97: 187$ & $4: 161$ & 41.9 & $1.2 \mathrm{e}-26^{* * *}$ \\
Cefoxitin & $9: 187$ & $1: 161$ & 8.1 & 0.12 \\
Cefopodoxim & $97: 187$ & $16: 161$ & 9.7 & $1.2 \mathrm{e}-16^{* * *}$ \\
Ceftazidim & $67: 187$ & $8: 161$ & 10.6 & $3.3 \mathrm{e}-12^{* * *}$ \\
Cefuroxim & $109: 187$ & $5: 161$ & 43.1 & $1.1 \mathrm{e}-30^{* * *}$ \\
Doripenem & $4: 187$ & $3: 161$ & 1.2 & 1 \\
Ertapenem & $4: 187$ & $2: 161$ & 1.7 & \\
Imipenem & $83: 187$ & $7: 161$ & 17.4 & $3.5 \mathrm{e}-18^{* * *}$ \\
Meropenem & $56: 186$ & $7: 161$ & 9.4 & $7.8 \mathrm{e}-10^{* * *}$ \\
Ciprofloxacin & $94: 187$ & $4: 161$ & 39.3 & $2.2 \mathrm{e}-25^{* * *}$ \\
Levofloxacin & $82: 187$ & $8: 161$ & 14.8 & $4.8 \mathrm{e}-17^{* * *}$ \\
Norfloxacin & $31: 187$ & $10: 161$ & 3.0 & $0.024^{*}$ \\
Amikacin & $136: 187$ & $24: 161$ & 15.1 & $2.2 \mathrm{e}-27^{* * *}$ \\
Gentamycin & $64: 187$ & $10: 161$ & 7.8 & $4.1 \mathrm{e}-10^{* * *}$ \\
Netilmicin & $15: 187$ & $4: 161$ & 3.4 & 0.13 \\
Tobramycin & $126: 187$ & $17: 161$ & 17.3 & $1.2 \mathrm{e}-27^{* * *}$ \\
Tigecyclin & $4: 187$ & $1: 161$ & 3.5 & 1 \\
Chloramphenicol & $128: 187$ & $39: 161$ & 6.7 & $1.4 \mathrm{e}-15^{* * *}$ \\
SXT & $52: 187$ & $25: 161$ & 2.1 & $0.046^{*}$ \\
\hline
\end{tabular}

Almost $90 \%$ of the E. coli isolates from airplane samples were resistant to at least one of the tested antibiotics (Fig. 5). This compares to about $45-60 \%$ in samples taken at WWTP. The peculiarity of airplane sewage is also reflected in the proportion of multiresistant isolates. Airplane-borne E. coli were far more likely to be resistant against $\geq 3$ classes of antibiotics than isolates obtained from raw sewage entering the WWTP (Fig. 5). 


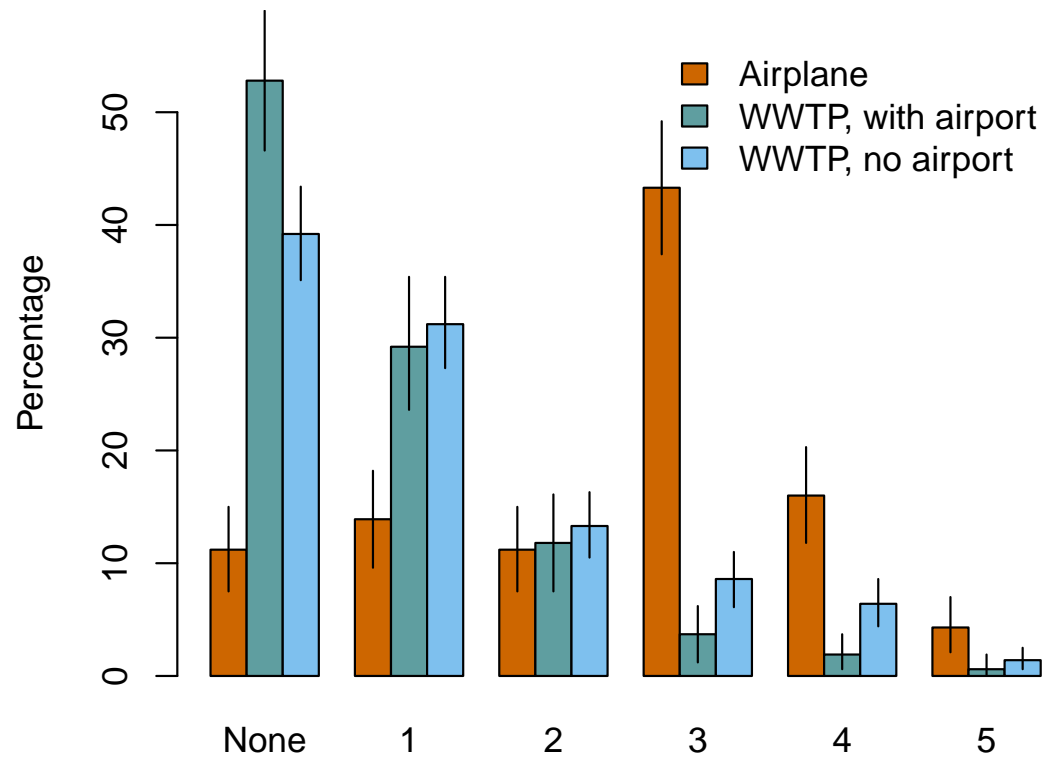

Figure 5: Prevalence of single- and multi-drug resistance among E. coli isolated from airplane sewage and WWTP influents with/without connection to airports. X-axis labels indicate the number of ineffective classes of drugs. The classes covered by susceptibility tests include penicillins, cephalosporines, carbapenemes, fluoroquionolones, aminoglycosides where each class is represented by $3-6$ antibiotics (see $1^{\text {st }}$ column of Table 3 ). Further drug classes represented by a single substance include tetracyclines, phenicols, and trimethoprim/sulfamethoxazole. Error bars represent $90 \%$ confidence intervals estimated by bootstrapping.

\subsection{Methodological aspects}

Since many of the available samples were processed in parallel through shotgun-sequencing and qPCR, there was a unique chance to compare the two approaches regarding the quantification of ARG. Doing so, we had to distinguish between unambiguous and potential hits in the metagenomes (Fig. 6). Unambiguous hits are those where a sample sequence ( $\approx 150 \mathrm{bp}$ ) matched just a single ARG registered in the data base (black symbols). This is in contrast to potential hits (gray symbols), where the sample sequence matched multiple related ARG among which is the particular gene of interest.

In general, we observed a reasonable agreement between the metagenomics and $\mathrm{qPCR}$ data for ARG with relative abundances greater than about $10^{-3}$ copies (16S rRNA gene

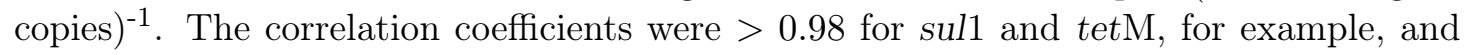
$>0.93$ for ermB (based on unambiguous hits). Nevertheless, Fig. 6 also shows some characteristic mismatches between the two approaches. Most notably, there appears to be a negative bias in the metagenomics-based estimates. At relative abundances $>0.01$ the deviation hardly exceeds $1 / 2 \log$ unit but underestimation gets stronger as relative abundances decline. That negative bias gradually turns into failure of the metagenomics approach (symbols accumulating on the lower axis) as the signatures of rare ARG occur just by chance in the set of sample sequences (about $5 \times 10^{7}$ in this study).

However, not all of the deviations depicted in Fig. 6 should be blamed on the limitations of metagenomics. For example, symbols associated with blaOXA-48 and blaTEM are far off the 1:1 line. This might well be due to unspecific amplification of primers 
resulting in an overestimation of ARG abundances by qPCR.

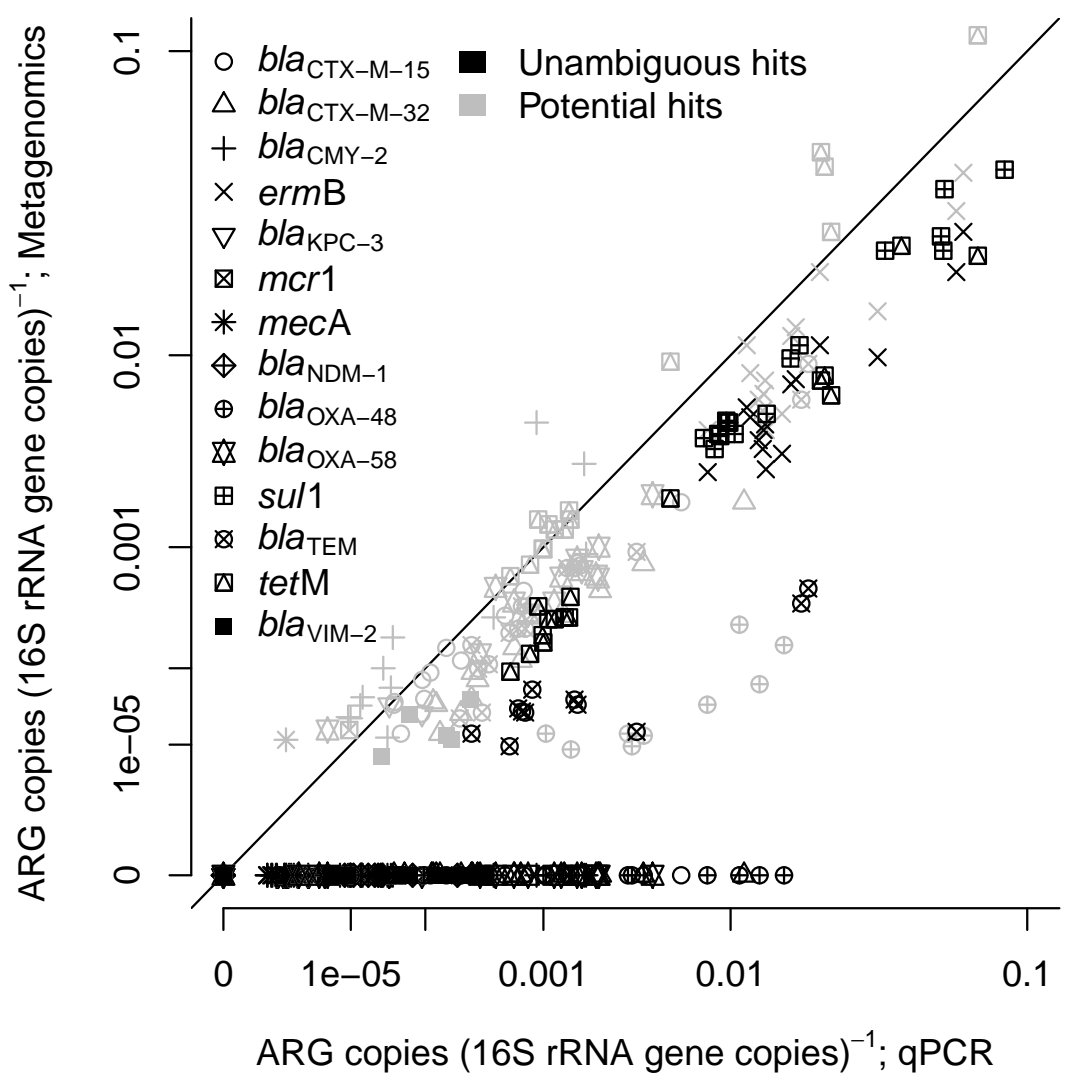

Figure 6: Comparison of relative ARG abundances obtained with qPCR and metagenomics for identical extracts of DNA. The diagonal indicates a 1:1 match. The data include raw sewage samples from all sources (airplane and WWTP). Note the custom axes resulting from square-root transformation.

\section{Discussion}

Cultivation and culture-independent techniques yielded a largely consistent and well differentiated picture of the prevalence of ARB/ARG in untreated wastewater of different origins. In agreement with initial expectations, sewage from airplane tanks was found to stand out from conventional sewage in terms of ARG diversity (Fig. 2). Nevertheless, the rarefaction models plotted in Fig. 3 suggest that the difference between airplane sewage and untreated municipal wastewater in terms of ARG diversity might level off for very large numbers of analyzed sequences. Considering the fact that municipal wastewater integrates bacteria from various sources besides those related to human waste (e.g. from pets, slaughterhouses, soil runoff, etc.) a convergence of the curves seems plausible. Thus, the main feature of airplane sewage appears to be the fact that a large number of different ARG can already be found in limited quantities of bacterial DNA. The mixing of human gut bacteria from different geographical backgrounds (hypothesis 1) provides a plausible explanation for this finding. Considerable variation in human resistomes is known to exist already at European level (European Centre for Disease Prevention and Control, 2017; Pärnänen et al., 2019) and even larger disparities are expected on global scales. For example, Bengtsson-Palme et al. (2015a) demonstrated the import of ESBL-producing E. coli by travelers returning from India while no such import was observed from Africa. However, the elevated diversity of ARG in airplane sewage could also be due to alternative 
mechanisms. For example, disparities in ARG diversity between samples of different origin might - at least in parts - reflect contrasts in taxonomic diversity. In particular, the elevated number of unique ARG in airplane sewage coincided with an increased diversity of bacterial groups as reflected in taxonomic evenness (Fig. 1) as well as in the number of bacterial families detected per $10^{5}$ DNA sequences (Sec. 3.1). Against this background, the true cause(s) of the increased ARG diversity in airplane-borne sewage are yet to be explored. Instead of focusing on ARG diversity, future research might also attempt to identify specific ARG which are characteristic for wastewaters of different origin. Our metagenomics-based data suggest that such characteristic genes exist. For example, the beta-lactamase gene bla $\mathrm{CARB}-4$ was found in high relative abundances in airplane sewage while it was never detected in any sample of municipal wastewater without contribution from airports. Similarly, the carbapenemase resistance gene blaOXA-427 was frequently detected in samples from WWTP influents while its signature was not found in any sample of airplane sewage.

Our data clearly support the second hypothesis according to which airplane-borne sewage stands out from common untreated municipal wastewater in terms of the relative abundance of ARG. The elevated prevalence of antibiotic resistance in airplane sewage was most clearly demonstrated by drug susceptibility tests carried out on E. coli (Table 3, Fig. 5). The high abundance of multi-resistant E. coli in airplane sewage is particularly remarkable. For example, the proportion of isolates carrying a combined resistance against $3^{\text {rd }}$ generation cephalosporins, fluorochinolones and aminoglycosides was increased by a factor of about eight compared to German clinical isolates (28.9\% compared to 3.5\%; European Centre for Disease Prevention and Control, 2017). With regard to the prevalence of genotypic resistance, the clearest differentiation between wastewaters of different origin was obtained by the metagenomics approach (Fig. 4). The median relative abundance of ARG was higher in airplane-borne sewage than in conventional sewage for seven out of nine classes of target drugs. Similar to the case of gene diversity, the elevated prevalence of ARG/ARB in airplane sewage might reflect differences in gut microbiomes between the local population (Germany) and the flights' or passengers' countries of origin. However, storage conditions in the airplanes' wastewater tanks provide an alternative explanation. Those tanks typically contain disinfectants. A common formulation certified for use in aircraft toilets lists alkylbenzyldimethylammonium chloride, a quaternary ammonium compound, as its major ingredient. A number of potential linkages between this compound and antibiotic resistance bacteria is known to exist (see SCENIHR, 2009, Sec. 3.8.5). The sewage tanks might thus serve as incubators that select for antibiotic resistance via mechanisms of, e.g, cross- or co-resistance (Buffet-Bataillon et al., 2012). The "breeding" of ARB should be particularly efficient if the tanks are not completely purged, leaving a highly resistant inoculum for continued vertical and horizontal ARG transfer.

In order to assess the potential risk associated with the special resistome of airplane sewage, information on the fate of the respective ARG/ARB is required. Our data indicate that airplane-borne sewage is strongly "diluted" upon mixing with wastewater from other sources. None of the employed methods (susceptibility tests, qPCR, metagenomics) indicated significant shifts in ARG diversity or ARG/ARB prevalence between samples of raw sewage acquired from WWTP with and without connection to airports. Assuming that bacteria and genes do not undergo considerable retention or degradation in the sewer system, that lack of significance should mainly reflect the limits of current analytical methods to detect small increments in ARG/ARB abundances in the presence of considerable background levels (unfavorable signal-to-noise ratio).

One might be tempted to conclude that airport-borne sewage is of little relevance for the dissemination of ARG because of the apparently strong dilution. Furthermore, 
airplane tanks are just one source of ARG and other hot-spots of antibiotic resistance are known to exist, e. g. large health care facilities. Such reasoning, however, disregards an essential property of genetic material, namely the potential for replication via horizontal and vertical transfer. Consequently, even very small amounts of ARG released into the water cycle may spread within the aquatic environment with the chance of (re)emerging in human pathogens. From this point of view, the potential threat coming from airport-borne sewage is not the quantity of imported ARG but the dissemination of resistances which are rarely found in the local environmental systems. This relates not only to rare ARG but also to combined resistances against multiple classes of drugs. With the example of E. coli, airplane-sewage was shown to be an unusual source of highly multi-resistant isolates. Nevertheless, we currently cannot provide evidence for the fact that rare ARG and/or combined resistances originating from airplane-sewage actually undergo considerable enrichment via selection or horizontal gene transfer in the receiving treatment plants. As pointed out by Bengtsson-Palme et al. (2016), comprehensive analyses of resistant and susceptible strains in WWTP influents and effluents would be necessary to specifically target that question. As a consequence of our study, we suggest to first explore evolutionary processes inside airplane waste tanks and to look for options that prevent (or deal with) the increased level of antibiotic resistance "begin-of-pipe".

\section{Acknowledgements}

A considerable part of the lab work was performed by our colleagues U. Mogck and C. Zschornack. The study has been funded by the German Federal Ministry of Education and Research [grant number 02WRS1377D] and the Graduate Academy of Technische Universität Dresden.

\section{Competing interests}

The authors declare no competing interests.

\section{References}

Alexander, J., Knopp, G., Dötsch, A., Wieland, A., and Schwartz, T. (2016). Ozone treatment of conditioned wastewater selects antibiotic resistance genes, opportunistic bacteria, and induce strong population shifts. Sci. Total Environ., 559:103-112.

Bengtsson-Palme, J., Angelin, M., Huss, M., Kjellqvist, S., Kristiansson, E., Palmgren, H., Larsson, D. G. J., and Johansson, A. (2015a). The human gut microbiome as a transporter of antibiotic resistance genes between continents. Antimicrobial Agents and Chemotherapy, 59(10):6551-6560.

Bengtsson-Palme, J., Hammarén, R., Pal, C., Östman, M., Björlenius, B., Flach, C., Fick, J., Kristiansson, E., Tysklind, M., and Larsson, D. (2016). Elucidating selection processes for antibiotic resistance in sewage treatment plants using metagenomics. Science of the Total Environment, 572:697-712.

Bengtsson-Palme, J., Hartmann, M., Eriksson, K. M., Pal, C., Thorell, K., Larsson, D. G. J., and Nilsson, R. H. (2015b). metaxa2: Improved identification and taxonomic classification of small and large subunit rRNA in metagenomic data. Molecular Ecology Resources, 15(6):1403-1414. 
Buffet-Bataillon, S., Tattevin, P., Bonnaure-Mallet, M., and Jolivet-Gougeon, A. (2012). Emergence of resistance to antibacterial agents: The role of quaternary ammonium compounds - a critical review. International Journal of Antimicrobial Agents, 39(5):381 -389 .

European Centre for Disease Prevention and Control (2017). Antimicrobial resistance surveillance in Europe 2016. Annual Report of the European Antimicrobial Resistance Surveillance Network (EARS-Net). ECDC, Stockholm.

European Surveillance of Antimicrobial Consumption Network (2017). Summary of the latest data on antibiotic consumption in the European Union; ESAC-Net surveillance data November 2017. ECDC, Stockholm.

Forslund, K., Sunagawa, S., Kultima, J. R., Mende, D. R., Arumugam, M., Typas, A., and Bork, P. (2013). Country-specific antibiotic use practices impact the human gut resistome. Genome Research, 23(7):1163-1169.

Hembach, N., Schmid, F., Alexander, J., Hiller, C., Rogall, E. T., and Schwartz, T. (2017). Occurrence of the mcr-1 colistin resistance gene and other clinically relevant antibiotic resistance genes in microbial populations at different municipal wastewater treatment plants in Germany. Frontiers in Microbiology, 8:1282.

Heß, S., Berendonk, T., and Kneis, D. (2018). Antibiotic resistant bacteria and resistance genes in the bottom sediment of a small stream and the potential impact of remobilization. FEMS Microbiology Ecology, 94:fiy128.

Holm, S. (1979). A simple sequentially rejective multiple test procedure. Scandinavian Journal of Statistics, 6(2):65-70.

Kurpiel, P. M. and Hanson, N. D. (2012). Point mutations in the inc antisense RNA gene are associated with increased plasmid copy number, expression of blaCMY-2 and resistance to piperacillin/tazobactam in Escherichia coli. The Journal of Antimicrobial Chemotherapy, 67(2):339-345.

Monteiro, J., Widen, R. H., Pignatari, A. C. C., Kubasek, C., and Silbert, S. (2012). Rapid detection of carbapenemase genes by multiplex real-time PCR. The Journal of Antimicrobial Chemotherapy, 67(4):906-909.

Nordmann, P., Poirel, L., Walsh, T. R., and Livermore, D. M. (2011). The emerging NDM carbapenemases. Trends in Microbiology, 19(12):588-595.

Osińka, A., Korzeniewska, E., Harnisz, M., and Niestępski, S. (2017). The prevalence and characterization of antibiotic-resistant and virulent Escherichia coli strains in the municipal wastewater system and their environmental fate. The Science of the Total Environment, 577:367-375.

Peak, N., Knapp, C. W., Yang, R. K., Hanfelt, M. M., Smith, M. S., Aga, D. S., and Graham, D. W. (2007). Abundance of six tetracycline resistance genes in wastewater lagoons at cattle feedlots with different antibiotic use strategies. Environmental Microbiology, 9(1):143-151.

Pärnänen, K., Narciso-da Rocha, C., Kneis, D., Berendonk, T., Cacace, D., Do, T., Elpers, C., Fatta-Kassinos, D., Henriques, I., Jaeger, T., Karkman, A., Martinez, J., Michael, S., Michael-Kordatou, I., O’Sullivan, K., Rodriguez-Mozaz, S., Schwartz, T., Sheng, H., Sørum, H., Stedtfeld, R., Tiedje, J., S.V., D., Walsh, F., Vaz-Moreira, I., Virta, M., and Manaia, C. (2019). Antibiotic resistance in European wastewater 
treatment plants mirrors the pattern of clinical antibiotic resistance prevalence. Science Advances, 5(3):eaau9124.

R Core Team (2017). R: A Language and Environment for Statistical Computing. R Foundation for Statistical Computing, Vienna, Austria.

Rosas, I., Salinas, E., Martínez, L., Cruz-Córdova, A., González-Pedrajo, B., Espinosa, N., and Amábile-Cuevas, C. F. (2015). Characterization of Escherichia coli isolates from an urban lake receiving water from a wastewater treatment plant in Mexico City: Fecal pollution and antibiotic resistance. Current Microbiology, 71(4):490-495.

SCENIHR (2009). Assessment of the antibiotic resistance effects of biocides. Scientific Committee on Emerging and Newly Identified Health Risks; European Commission, Health and Consumer Protection DG.

Stoddard, S. F., Smith, B. J., Hein, R., Roller, B. R., and Schmidt, T. M. (2015). rrnDB: improved tools for interpreting rRNA gene abundance in bacteria and archaea and a new foundation for future development. Nucleic Acids Research, 43(Database issue):D593-D598.

Szczepanowski, R., Linke, B., Krahn, I., Gartemann, K.-H., Gützkow, T., Eichler, W., Pühler, A., and Schlüter, A. (2009). Detection of 140 clinically relevant antibioticresistance genes in the plasmid metagenome of wastewater treatment plant bacteria showing reduced susceptibility to selected antibiotics. Microbiology, 155(Pt 7):23062319 .

Van Boeckel, T. P., Gandra, S., Ashok, A., Caudron, Q., Grenfell, B. T., Levin, S. A., and Laxminarayan, R. (2014). Global antibiotic consumption 2000 to 2010: an analysis of national pharmaceutical sales data. The Lancet Infectious Diseases, 14(8):742-750.

van der Zee, A., Roorda, L., Bosman, G., Fluit, A., Hermans, M., Smits, P., van der Zanden, A., Te Witt, R., Bruijnesteijn van Coppenraet, L., Cohen Stuart, J., and Ossewaarde, J. (2014). Multi-centre evaluation of real-time multiplex PCR for detection of carbapenemase genes OXA-48, VIM, IMP, NDM and KPC. BMC Infectious Diseases, 14:27.

Volkmann, H., Schwartz, T., Bischoff, P., and Kirchen, S. andObst, U. (2004). Detection of clinically relevant antibiotic-resistance genes in municipal wastewater using real-time PCR (TaqMan). Journal of Microbiological Methods, 56(2):277-286.

World Health Organization (2014). Antimicrobial resistance: Global report on surveillance 2014. ISBN: 9789241564748 .

World Health Organization (2015). Global action plan on antimicrobial resistance. ISBN: 9789241509763.

Zankari, E., Hasman, H., Cosentino, S., Vestergaard, M., Rasmussen, S., Lund, O., Aarestrup, F. M., and Larsen, M. V. (2012). Identification of acquired antimicrobial resistance genes. Journal of Antimicrobial Chemotherapy, 67(11):2640-2644. 
${ }_{500}$ Supplement 


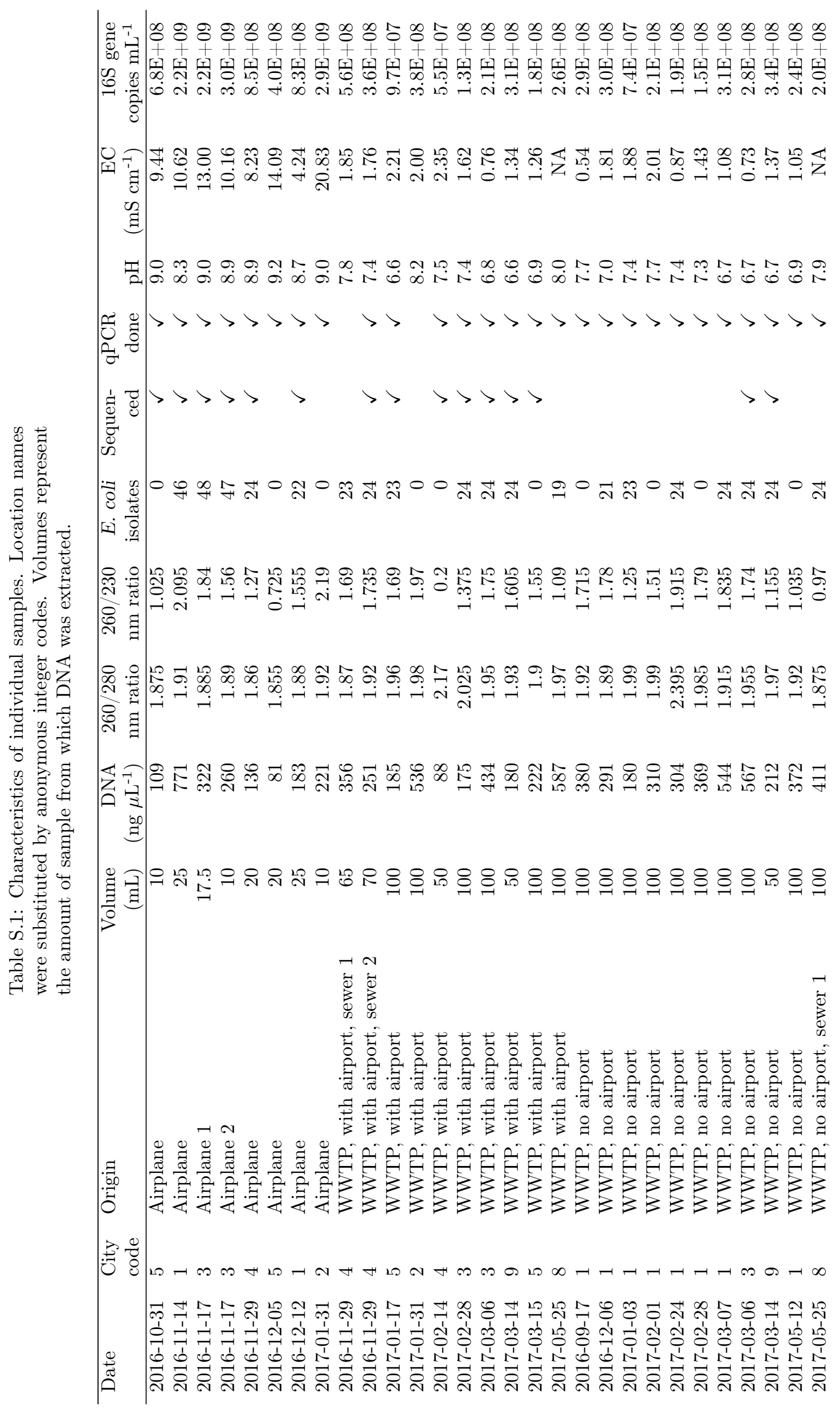




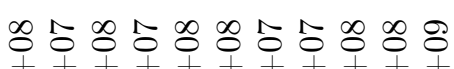

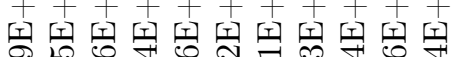
ง

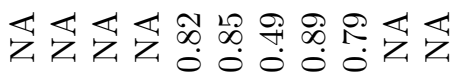

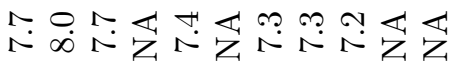
$>>>>>>>$

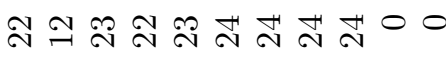

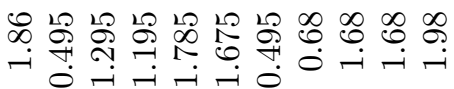

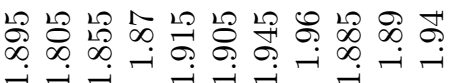

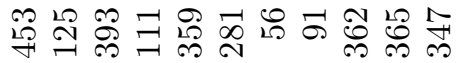

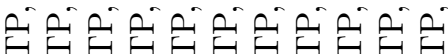
管 $\infty \infty \infty \cup \neg \neg \neg \wedge 0 \wedge 0$

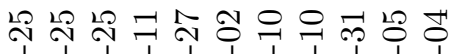

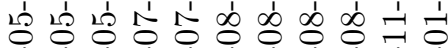

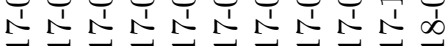

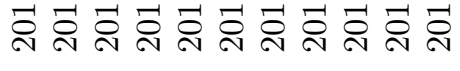


Table S.2: List of qPCR primers.

\begin{tabular}{|c|c|c|c|c|}
\hline Gene & & Primer sequence & $\begin{array}{l}\text { Amplicon } \\
\text { size (bp) }\end{array}$ & Reference \\
\hline \multirow[t]{2}{*}{ blaTEM } & Fwd. & TTCCTGTTTTTGCTCACCCAG & 112 & NORMAN Network $^{1}$ \\
\hline & Rev. & CTCAAGGATCTTACCGCTGTTG & & \\
\hline \multirow[t]{2}{*}{ ermB } & Fwd. & TGAATCGAGACTTGAGTGTGCAA & 71 & Alexander et al. (2016) \\
\hline & Rev. & GGATTCTACAAGCGTACCTT & & \\
\hline \multirow[t]{2}{*}{ tetM } & Fwd. & GGTTTCTCTTGGATACTTAAATCAATCR & 88 & Peak et al. (2007) \\
\hline & Rev. & CCAACCATAYAATCCTTGTTCRC & & \\
\hline \multirow[t]{2}{*}{ sul1 } & Fwd. & CGCACCGGAAACATCGCTGCAC & 161 & NORMAN Network $^{1}$ \\
\hline & Rev. & TGAAGTTCCGCCGCAAGGCTCG & & \\
\hline \multirow[t]{2}{*}{ bla $\mathrm{CMY-2}$} & Fwd. & CGTTAATCGCACCATCACC & 172 & Kurpiel and Hanson (2012) \\
\hline & Rev. & CGTCTTACTAACCGATCCTAGC & & \\
\hline \multirow[t]{2}{*}{ bla } & Fwd. & CTATGGCACCACCAACGATACTYM & 103 & ANSWER Network ${ }^{2}$ \\
\hline & Rev. & ACGGCTTTCTGCCTTAGGTT & & \\
\hline \multirow[t]{2}{*}{ bla } & Fwd. & CGTCACGCTGTTGTTAGGAA & 155 & NORMAN Network $^{1}$ \\
\hline & Rev. & CGCTCATCAGCACGATAAAG & & \\
\hline \multirow[t]{2}{*}{ bla OXA-48 } & Fwd. & TGTTTTTGGTGGCATCGAT & 177 & Monteiro et al. (2012) \\
\hline & Rev. & GTAAMRATGCTTGGTTCGC & & \\
\hline \multirow[t]{2}{*}{ bla OXA-58 } & Fwd. & GCAATTGCCTTTTAAACCTGA & 152 & Szczepanowski et al. (2009) \\
\hline & Rev. & CTGCCTTTTCAACAAAACCC & & \\
\hline \multirow[t]{2}{*}{ mecA } & Fwd. & CGCAACGTTCAATTTAATTTTGTTAA & 91 & Volkmann et al. (2004) \\
\hline & Rev. & TGGTCTTTCTGCATTCCTGGA & & \\
\hline \multirow[t]{2}{*}{ bla $a_{\mathrm{NDM}-1}$} & Fwd. & TTGGCCTTGCTGTCCTTG & 82 & Monteiro et al. (2012) \\
\hline & Rev. & ACACCAGTGACAATATCACCG & & \\
\hline \multirow[t]{3}{*}{ blaviM-2 } & Fwd. & GAGATTCCCACGCACTCTCTAGA & 93 & van der Zee et al. (2014) \\
\hline & Rev. & AATGCGCAGCACCAGGATAG & & \\
\hline & probe & ACGCAGTGCGCTTCGGTCCAGT & & \\
\hline \multirow[t]{2}{*}{ bla $\mathrm{KPC}_{-3}$} & Fwd. & CAGCTCATTCAAGGGCTTTC & 196 & Szczepanowski et al. (2009) \\
\hline & Rev. & GGCGGCGTTATCACTGTATT & & \\
\hline \multirow[t]{2}{*}{ mcr 1} & Fwd. & GGGCCTGCGTATTTTAAGCG & 183 & Hembach et al. (2017) \\
\hline & Rev. & CATAGGCATTGCTGTGCGTC & & \\
\hline \multirow[t]{2}{*}{ 16S rRNA } & Fwd. & TCCTACGGGAGGCAGCAGT & 195 & NORMAN Network ${ }^{1}$ \\
\hline & Rev. & ATTACCGCGGCTGCTGG & & \\
\hline
\end{tabular}

$1_{\text {WWW . norman-network.net }}$

${ }^{2}$ www . answer-itn. eu 